\title{
Elements of a Purpose Built Electron Cryomicroscope for Single-particle CryoEM
}

\author{
Christopher Russo and Richard Henderson
}

MRC Laboratory of Molecular Biology, Cambridge, England, United Kingdom

Single-particle electron cryomicroscopy (cryoEM) might soon overtake X-ray crystallography as the most common method of protein structure determination (Fig. 1, number of structures deposited per method per year, the Protein Data Bank, pdb.org). Progress has come from improvements in the microscope vacuum, electron gun, detectors (direct electron, introduced in 2012), specimen supports, preparation methods and new algorithms and software. Single-particle analysis with electrons is less demanding on sample quantity and purity, and avoids the trial and error with large amounts of purified material required for crystallisation. State of the art electron cryomicroscopes from current commercial vendors have a 300 $\mathrm{keV}$ electron source and a total operational cost of order $£ 10 \mathrm{M}$ over 10 years. Recent work has shown that three problems thought to prevent low-energy cryoEM - charge build-up, microscopic charge fluctuations, and Ewald sphere curvature - do not produce significant quantitative image degradation [1-3]. Further, we have shown that lowering the electron energy gives images that contain more information for the same amount of radiation damage. The optimum electron energy for single particle analysis of a $300 \AA$ thick, plunge-frozen, amorphous-ice specimen is $100 \mathrm{keV}$ [4], and this energy is practical for high-resolution structure determination for a range of specimens [5].

Given these studies, we can now fully describe the requirements of a purpose-built electron cryomicroscope for single-particle cryoEM, that maximises the potential information in each electron cryomicrograph whilst minimising the total cost of ownership (Fig. 2). A $100 \mathrm{keV}$ cryoEM is as capable but simpler and less expensive than a $300 \mathrm{keV}$ cryoEM (order 10-fold reduction in cost over 10 years). In developing this design we consider the various physical phenomenon that limit cryoEM currently, and specify the hardware requirements that are essential for a low-cost, high-resolution electron cryomicroscope. These include novel electron sources, stages, lenses, anti-contamination systems and detectors - currently in development - that are specifically designed for the needs of single-particle cryoEM. Various tradeoffs like ultimate resolution vs. cost and potential future improvements in hardware will also be described. 


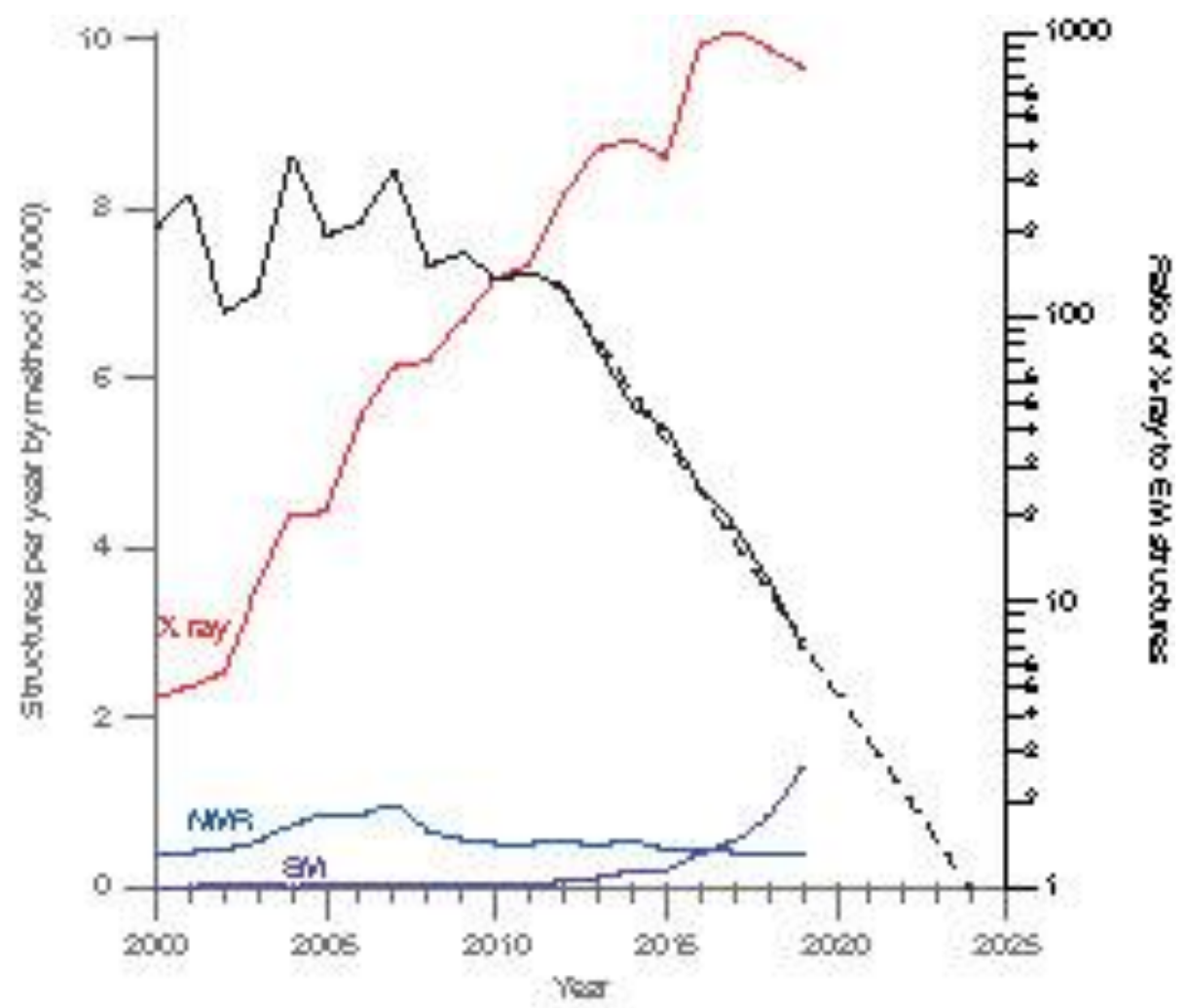

Figure 1. Atomic structures deposited in the protein data bank (PDB.org) by method per year. Dotted line indicates the exponential growth in structures determined by electron cryomicroscopy, beginning with the introduction of direct electron detectors in 2012.

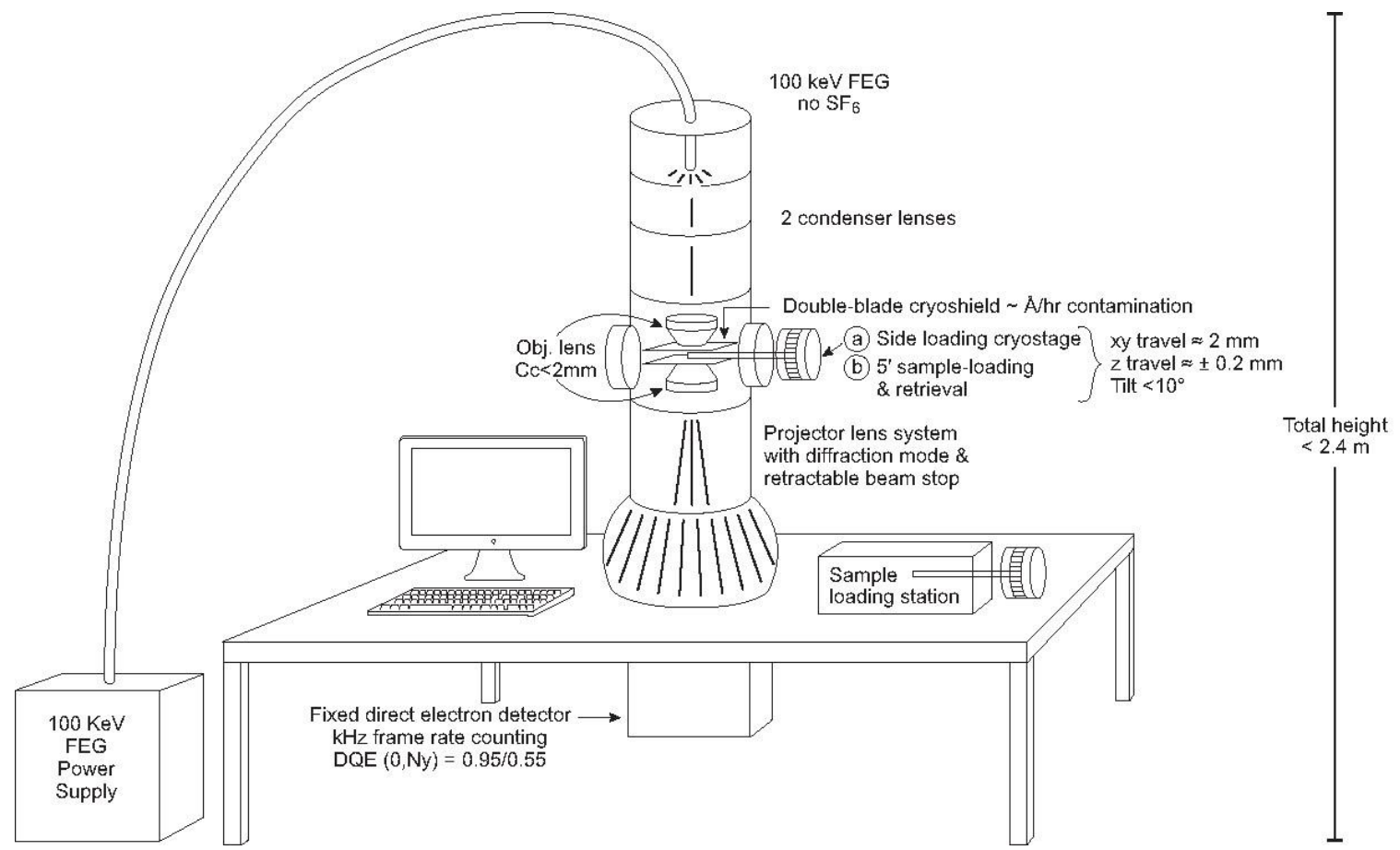

Figure 2. Diagram of a purpose built electron cryomicroscope for low dose cryoEM of biological specimens. 


\section{References}

1. C.J. Russo and R. Henderson "Charge accumulation in electron cryomicroscopy" Ultramicroscopy 187 , 43-49 (2018).

2. C.J. Russo and R. Henderson "Microscopic charge fluctuations cause minimal contrast loss in cryoEM" Ultramicroscopy 187, 56-63 (2018).

3. C.J. Russo and R. Henderson "Ewald sphere correction using a single side-band image processing algorithm" Ultramicroscopy 187, 26-33 (2018).

4. M.J. Peet, R. Henderson \& C.J. Russo "The energy dependence of contrast and damage in electron cryomicroscopy of biological molecules" Ultramicroscopy 203, 125-131 (2019).

5. K. Naydenova, G. McMullan, M.J. Peet, Y. Lee, P.C. Edwards, S. Chen, E. Leahy, S. Scotcher, R. Henderson, C.J. Russo "CryoEM at $100 \mathrm{keV}$ : a demonstration and prospects" IUCrJ 6, 1086-1098 (2019). 